\title{
Metaraminol and Noradrenaline in Septic Shock: A Retrospective Comparison
}

Anirudh Krishnamohan ( $\nabla$ anirudh.krishnamohan@health.nsw.gov.au )

Royal North Shore Hospital https://orcid.org/0000-0002-5010-3716

Anthony Delaney

RNSH: Royal North Shore Hospital

Mark Gillett

Royal North Shore Hospital

\section{Research}

Keywords: Metaraminol, Noradrenaline, Sepsis, Shock, Infection, Vasopressor, Inotrope

Posted Date: September 30th, 2021

DOI: https://doi.org/10.21203/rs.3.rs-934176/v1

License: (c) (i) This work is licensed under a Creative Commons Attribution 4.0 International License. Read Full License 


\section{Abstract}

Introduction

Vasopressor use is an important facet of septic shock management, in order to maintain hemodynamic targets and end organ perfusion. Traditionally, Noradrenaline has been the 'gold standard' drug of choice for septic shock. Metaraminol is an alternative vasopressor that has been used for septic shock. However, there has been minimal research in comparing the two drugs in septic patients, particularly with regards to total time spent on infusion.

Objectives

To compare total time spent on either Metaraminol or Noradrenaline infusion by septic shock patients, whilst adjusting for baseline severity of illness. Secondary outcomes included incidence of mechanical ventilation and new requirement of renal replacement therapy, and mortality.

Methods

A retrospective medical records review was undertaken, looking at all septic shock patients admitted to ICU in 2019, who received either Metaraminol or Noradrenaline. Data extracted from eRIC (the ICU database) included total time spent on infusion, APACHE III scores, incidence of mechanical ventilation, incidence of renal replacement therapy, and mortality.

Results

Our review yielded 174 patients who were eligible for further statistical analysis (63 in Metaraminol group, and 111 in the Noradrenaline group). The mean duration of infusion in the Metaraminol group was 1655 minutes, and 2663 minutes in the Noradrenaline group. The mean APACHE III Scores were 62 in the Metaraminol group and 77 in the Noradrenaline group. A one-way ANCOVA test found that there was a statistically significant $[F(1,171)=4.511, p=0.035]$ reduction in time spent on Metaraminol infusion, compared with Noradrenaline, after adjusting for baseline severity of illness by way of APACHE III Score.

Conclusion

Our study found a statistically significant reduction in time spent on a Metaraminol infusion compared with Noradrenaline by septic shock patients, after controlling for severity of illness. However, due to its retrospective study design, we were unable to account for bias and confounders, such as antibiotic and fluid administration, or clinician preference for one drug over the other. Nevertheless, our study adds to the paucity of literature comparing Metaraminol to Noradrenaline, and paves the way for future randomized trials comparing the two drugs in septic shock.

\section{Introduction}


Sepsis accounts for a significant burden of disease, with worldwide cases up to 50 million a year, including 11 million deaths (1). Left untreated, sepsis can rapidly lead to multi-organ failure and death, due to a dysregulated host immune response to infection. Current resuscitation guidelines include early broad spectrum antibiotic administration and infection source control, along with aggressive fluid resuscitation to avoid developing septic shock. For those in shock, vasopressors are recommended to maintain organ perfusion and avoid progression to multi-organ failure and death (2).

The most common vasopressor used in septic shock is Noradrenaline $(2,13)$, a catecholamine with predominant alpha ${ }_{1}$ activity, and beta ${ }_{1}$ to a lesser extent (3). Metaraminol is a pure alpha ${ }_{1}$ agonist, but indirectly leads to endogenous Noradrenaline release (3). Both increase peripheral vascular resistance and mean arterial pressure, thereby improving organ perfusion. Several studies have looked at Noradrenaline in septic shock, however similar research on Metaraminol is scarce (4-7).

Metaraminol has been used for septic shock since the 1960s (11), but despite this, there is a paucity of high level research on its use in sepsis, particularly in comparison with Noradrenaline. Existing research includes anecdotal case reports and small observational studies, but none comparing Metaraminol directly with Noradrenaline. Both agents are used around the world for septic shock, with the choice of agent depending largely on clinician preference or institutional policy (3).

This study aims to investigate the association between choice of vasopressor (Metaraminol or Noradrenaline) and total duration of vasopressor support (in minutes), in septic shock patients. Secondary outcomes include incidence of mechanical ventilation and new requirement of renal replacement therapy (RRT), surrogate markers of end organ perfusion. Other secondary markers include total ICU length of stay (LOS), and mortality.

\section{Methods}

Study design

Approval was granted by the Northern Sydney Local Health District Human Research Ethics Committee (2021/PID00874) for a single centre retrospective medical records review.

Setting and participants

Patients were retrospectively recruited from a quaternary metropolitan Australian ICU's database (eRIC). A list was compiled of patients admitted to the ICU in 2019, along with their ANZICS diagnosis codes, age, gender, source of admission, as well as relevant data relating to primary and secondary outcomes (e.g. receiving either Metaraminol or Noradrenaline, requiring mechanical ventilation or renal replacement therapy).

The ANZICS diagnosis codes were analysed for specific keywords relating to infection, such as 'sepsis', 'pneumonia', 'abscess'. Patients were enrolled based on these keywords, indicating infection from any 
source. A table of the ANZICS diagnosis codes which were used to capture and enrol patients is attached as Appendix 1. Patients under the age of 18 were excluded.

\section{Outcomes}

The primary outcome was total time spent on either a Metaraminol or Noradrenaline infusion. Secondary outcomes included ICU LOS, mortality, and incidence of mechanical ventilation and renal replacement therapy.

\section{Confounders and bias}

Due to a retrospective study design, it was not possible to control for confounders. Vasopressor support is only one facet of sepsis management, with adequate fluid resuscitation, appropriate antibiotic therapy and source control, and (in some cases) metabolic resuscitation via administration of steroids, all contributing to halting disease progression. Data on these variables (such as timing of antibiotics, amount of fluids given, and/or steroid administration) was not readily available for extraction and analysis from eRIC.

Inherent bias may be present in the study due to clinician preference for one particular vasopressor over the other. Traditionally, the 'sicker' septic patients received Noradrenaline, whilst Metaraminol has been used as bridging until a central line can be placed. The reasoning behind vasopressor choice was not readily available from the eRIC database, and therefore could not be used to rationalise bias over one or the other drug.

\section{Statistical analysis}

Statistical analysis was performed using the one-way ANCOVA test to compare Metaraminol and Noradrenaline infusion times, whilst controlling for illness severity via the APACHE III scores. This particular test was chosen as it would allow us to compare the effect of a categorical variable (choice of vasopressor) on a continuous variable (time spent on infusion), whilst controlling for another continuous variable (APACHE III score as a surrogate for severity of illness). Using this test, we were able to directly compare Metaraminol with Noradrenaline, whilst controlling for baseline severity of illness.

\section{Results}

Review of the eRIC database for 2019 yielded 3767 patients. 251 patients received Metaraminol or Noradrenaline (or both), and had an ANZICS admission diagnosis code which included 'sepsis, infection, abscess' or system specific diagnosis of infection (e.g. cholangitis, cellulitis, pneumonia). Of these patients, 75 patients received both Metaraminol and Noradrenaline during their ICU admission, and therefore removed from analysis. The remaining 176 patients were included in the analysis as per the inclusion criteria; over 18 years of age and requiring either Metaraminol or Noradrenaline for septic shock. 64 of these patients received Metaraminol and 112 received Noradrenaline. 
One outlier was removed from each group. In the Noradrenaline group, one patient received Noradrenaline for only one minute, without ongoing infusion of either Noradrenaline or Metaraminol. After a review of the patient's record, it was deemed that they in fact did not receive any vasopressor, and were not septic. In the Metaraminol group, one patient received Metaraminol for 12802 minutes (the longest duration in the Metaraminol group). On review of the patient's record, it was noted that the patient had chronic asymptomatic hypotension, with a negative septic screen. It was deemed that the patient's hypotension was physiological, rather than secondary to sepsis.

This left 63 patients in the Metaraminol group and 111 patients in the Noradrenaline group, who underwent statistical analysis.

The one-way ANCOVA test found that there was a statistically significant $[F(1,171)=4.511, p=0.035]$ reduction in time spent on Metaraminol infusion than Noradrenaline, after accounting for illness severity. The large sample size allowed for analysis of a non-normal distribution of data, and Levene's Test showed equal variance between the groups. As we were comparing only two groups, post-hoc analyses were not required. 
Table 1

Patient baseline and demographic characteristics

\begin{tabular}{|lll|}
\hline Characteristic & $\begin{array}{l}\text { Metaraminol } \\
(\mathbf{n}=63)\end{array}$ & Noradrenaline $(\mathbf{n}=\mathbf{1 1 1})$ \\
\hline Median Age (years) & $71(18-91)$ & $65(18-91)$ \\
\hline Number female (\%) & $27(43 \%)$ & $54(49 \%)$ \\
\hline Mean APACHE III Score & $62(27-94)$ & $77(29-176)$ \\
\hline Outcomes & & \\
\hline Mean duration of infusion (min) & $1655(51-8169)$ & $2663(26-21892)$ \\
\hline Mean Length of Stay (hour) & $80(9-244)$ & $135(2-609)$ \\
\hline Ventilated & $9(14 \%)$ & $31(28 \%)$ \\
\hline CRRT & $0(0 \%)$ & $5(4 \%)$ \\
\hline Both & $0(0 \%)$ & $11(10 \%)$ \\
\hline Died in ICU & $3(5 \%)$ & $13(12 \%)$ \\
\hline Died in Hospital & $2(3 \%)$ & $3(3 \%)$ \\
\hline Source & & $8(7 \%)$ \\
\hline Uncoded & $22(35 \%)$ & $52(47 \%)$ \\
\hline Endocarditis & $0(0 \%)$ & $3(3 \%)$ \\
\hline Respiratory & $21(33 \%)$ & $20(18 \%)$ \\
\hline Gastrointestinal & $11(17 \%)$ & $22(20 \%)$ \\
\hline Urinary & $4(6 \%)$ & $6(5 \%)$ \\
\hline Gynaecological & $2(5 \%)$ & \\
\hline Soft tissue & & \\
\hline
\end{tabular}

\section{Discussion}

This study was a retrospective records review of septic shock patients admitted to a single metropolitan ICU in 2019. We found that despite a higher mean APACHE III score and higher incidence of mechanical ventilation and RRT in the Noradrenaline group, there was a statistically significant reduction (after adjusting for severity of illness) in time spent on vasopressor in patients receiving Metaraminol, as opposed to those receiving Noradrenaline. 
There have been previous studies comparing various inotropes/vasopressors, including Noradrenaline, Adrenaline, and Vasopressin, but only very few that included Metaraminol (3-8). This is the first study till date as far as the authors are aware, that directly compares Metaraminol to Noradrenaline in septic shock.

There were several drawbacks to this study. Due to its retrospective design, there was lack of control over confounders and variables. We could not feasibly collect data on confounders to duration of vasopressor support, such as fluid resuscitation, timing of antibiotics and/or source control, and provision of steroids for septic shock. These factors may have had an impact on the total duration of vasopressor support. The sample size for this study was relatively small, as it only included patients from one calendar year (2019). There were also double the number of patients in the Noradrenaline group as opposed to Metaraminol. This may have been due to clinical preference, as Noradrenaline is currently considered the 'gold standard' inotropic support in shock. Using the APACHE III score as a marker of illness severity may exclude other causes of sickness not calculated by the score. Therefore, there may have been sicker patients in the Noradrenaline group despite adjusting for the APACHE III scores, as evidenced by the higher incidence of ventilation and renal replacement. This could provide a reasoning as to why patients in the Noradrenaline group spent longer on an infusion, than those on Metaraminol. Seventy-five patients received both Metaraminol and Noradrenaline. These patients were excluded from the final analysis, as it was impossible to compare outcomes due to the use of both drugs. It is also difficult to speculate the cause for this, without review of each individual patient's record.

\section{Conclusion}

Despite its drawbacks, our study is the first of its kind (to our knowledge) to compare Metaraminol and Noradrenaline use in septic shock, and found that there was a statistically significant reduction in time spent on Metaraminol than Noradrenaline, in this cohort of patients. There is not enough evidence to postulate causations, but it is hypothesis generating, paving the way for future research in this area.

Currently, there is scarce evidence in current literature comparing both drugs in septic shock, and certainly no randomised trials. Future randomised trials with sound methodology designed to control for bias and confounders are warranted based on these results.

\section{Declarations}

\section{Ethics approval}

Approval was granted by the Northern Sydney Local Health District Human Research Ethics Committee (2021/PID 00874) for a single centre retrospective medical records review.

\section{Consent for publication}

Not applicable 


\section{Availability of data and materials}

Not applicable

\section{Competing interests}

The authors declare that they have no competing interests.

\section{Funding}

This study was funded via the Emergency Department's Research Unit at Royal North Shore Hospital. No external funding was received.

\section{Authors' contribution}

AK conceived the research question and conducted literature review, data and statistical analysis, and manuscript write up. $A D$ and $M G$ assisted with draft revisions and editing, suggestions, and advice. All authors read and approved the final manuscript.

\section{Acknowledgements}

The authors would like to sincerely thank Phil Johnson and Katelyn Davis (ICU Data Managers) for their patience and assistance with extracting and collating the necessary data from the ICU database.

\section{References}

1. Rudd KE, Johnson SC, Agesa KM, Shackelford KA, Tsoi D, Kievlan DR, et al. Global, regional, and national sepsis incidence and mortality, 1990-2017: analysis for the Global Burden of Disease Study. The Lancet. 2020;395(10219):200-11.

2. Rhodes A, Evans LE, Alhazzani W, Levy MM, Antonelli M, Ferrer R, et al. Surviving Sepsis Campaign: International Guidelines for Management of Sepsis and Septic Shock: 2016. Intensive Care Med. 2017;43(3):304-77.

3. Senz A, Nunnink L. Review article: inotrope and vasopressor use in the emergency department. Emerg Med Australas. 2009;21(5):342-51.

4. Myburgh JA, Higgins A, Jovanovska A, Lipman J, Ramakrishnan N, Santamaria J, et al. A comparison of epinephrine and norepinephrine in critically ill patients. Intensive Care Med. 2008;34(12):2226-34. 
5. Natalini G, Schivalocchi V, Rosano A, Taranto M, Pletti C, Bernardini A. Norepinephrine and Metaraminol in septic shock: a comparison of the hemodynamic effects. Intensive Care Med. 2005;31(5):634-7.

6. Russell JA. Vasopressor therapy in critically ill patients with shock. Intensive Care Med. 2019;45(11):1503-17.

7. Investigators A, Group ACT, Peake SL, Delaney A, Bailey M, Bellomo R, et al. Goal-directed resuscitation for patients with early septic shock. N Engl J Med. 2014;371(16):1496-506.

8. Anderson $\mathrm{KCH}$. BET 3: Peripheral Metaraminol Infusion in the Emergency Department. Emerg Med J. 2017;34(3):190-2.

9. Delaney A, Finnis M, Bellomo R, Udy A, Jones D, Keijzers G, et al. Initiation of vasopressor infusions via peripheral versus central access in patients with early septic shock: A retrospective cohort study. Emerg Med Australas. 2020;32(2):210-9.

10. Avni T, Lador A, Lev S, Leibovici L, Paul M, Grossman A. Vasopressors for the Treatment of Septic Shock: Systematic Review and Meta-Analysis. PLoS One. 2015;10(8):e0129305.

11. Cavanagh D, McLeod A. Septic shock in Obstetrics and Gynaecology; An evaluation of Metaraminol therapy. American Journal of. 1966;96(7):913-8.

12. Richardson D. Increase in patient mortality at 10 days associated with emergency department overcrowding. The Medical Journal of Australia. 2006;184(5):213-6.

13. Vail E, Gershengorn HB, Hua M, Walkey AJ, Rubenfeld G, Wunsch H. Association Between US Norepinephrine Shortage and Mortality Among Patients With Septic Shock. JAMA. 2017;317(14):1433-42.

\section{Figures}


1717 patients received Metaraminol and/or Noradrenaline

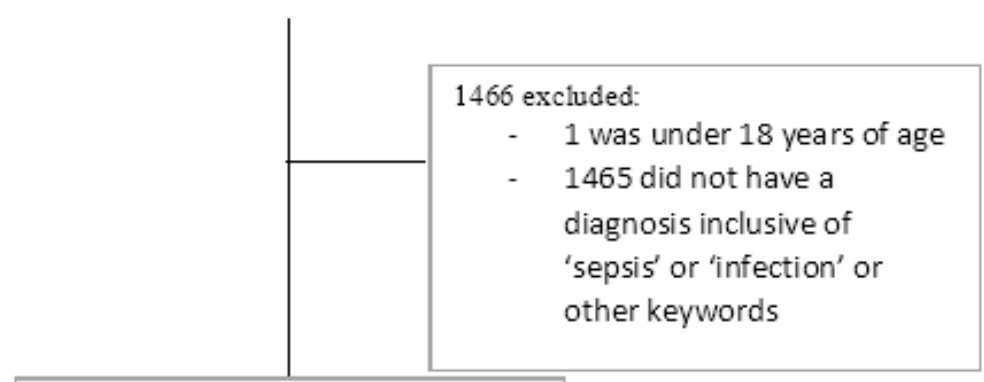

251 patients with a diagnosis of sepsis (or infection) needing Metaraminol and/or Noradrenaline

64 received Metaramino1

1 outlier excluded

\section{5 excluded for receiving both}

176 with sepsis/infection receiving only either Metaraminol or Noradrenaline

112 received Noradrenaline

1 outlier excluded

\section{Figure 1}

Patient recruitment

\section{Supplementary Files}

This is a list of supplementary files associated with this preprint. Click to download. 
- Appendix.docx

Page 11/11 